\title{
Optimal Design of Yagi-Udi Antenna for VHF Band Application Using Magus 2.2 Software
}

\author{
Goshwe, N. Y. and Tijam, I. P. \\ Department of Electrical/Electronics Engineering \\ University of Agriculture, Makurdi, Nigeria \\ Contact: nentawe@gmail.com
}

\begin{abstract}
This paper presents an optimal design of low cost Yagi Udi antennas. The design targets the field strength in the main beam to be larger than a critical minimum value, the directivity to be large enough over the whole frequency band, the antenna's input impedance to match the generator reasonably well over the entire band and that the VSWR be small. Nigerian Television Authority, Makurdi Network centre in Nigeria parameters was used. It transmits at VHF, operating on Channel 13 (215 - 218) MHz. The specification was used to calculate values for the antenna dimensions to be used for the simulation. Antenna Magus 2.2 software was used to simulate the optimal designed conditions of the Yagi-Uda antenna. For this design, the director spacing of the designed antenna was varied while maintaining the spacing between the first director and keeping the driven element constant. Optimal design was obtained at a gain of $14.37 \mathrm{dBi}$ and frequency of $223.7 \mathrm{MHz}$. Peak real impedance of the antenna was attained at $134.61 \Omega$ with a corresponding frequency of $259.7 \mathrm{MHz}$ and the Real impedance at zero-crossing frequency increased to $334.0 \Omega$ at Frequency $200.9 \mathrm{MHz}$. The VSWR of as low as 1.463 was obtained at frequency of $212.8 \mathrm{MHz}$, which falls within the VHF operating frequency. These results show a significant reduction in the value of VSWR and increase in both gain and bandwidth.
\end{abstract}

\section{INTRODUCTION}

Antenna is a transitional structure between free space and a guiding device that is made to efficiently radiate and receive radiated electromagnetic waves. Antennas are commonly used in radio, television broadcasting, cell phones, radar and other systems involving the use of electromagnetic waves. Antennas demonstrate a property known as reciprocity, which means that an antenna will maintain the same characteristics whether it is transmitting or receiving (Gary 1996). One of the applications of a one way wireless communication which antennas play a key role is the terrestrial television (TV). In terrestrial TV system, the transmitters (broadcast stations) are transmitting the TV signal with high power and very tall transmitting antenna located on the ground to transmit radio waves to the surrounding area (Bemani and Nikmehr, 2009). The choice of a particular antenna depends on factors such as gain, radiation pattern, polarization, bandwidth, resonant frequency and impedance.

The most common antenna used for receiving TV signals is the Yagi-Uda antenna (variation of the dipole antenna) which is relatively inexpensive to manufacture and design because of the simple 2- dimensional physical geometry (Cheng, 1991). They are usually employed at VHF and higher frequencies (VF) because the size of the antenna is directly tied to the wavelength at the resonant frequency. The Yagi antenna not only has unidirectional radiation and response pattern, but it concentrates the radiation and response (Barclay, L., 1997, Sun et al 2010). The main challenge posed by this type of antenna is because it presents difficult design and optimization challenges (Cheng, 1991, Lohn et al 2001, Goudos et al 2010).

Antenna Theory: The main theory of antennas covers directivity, radiation pattern, gain, mutual impedances between linear elements and coupling in receiving modes.

Directivity: Directivity describes how much energy is concentrated in one direction in preference to radiation in other directions. It is defined as the ratio of the radiation intensity in a certain direction to the average radiation intensity (Perambur and Rajeswari, 2003). It is given as

$D(\theta, \phi)=\frac{U(\theta, \phi)}{U_{\text {ave }}}$ 
Where $U(\theta, \phi)$ is the radiation intensity of the antenna while $U_{\text {ave }}$ is the average radiation.

Directivity can also be defined as a ratio of power density in a certain range by simply dividing the numerator and denominator by square of range $\left(r^{2}\right)$. By multiplying the power density by the square of the radius $r$ at which it is measured, we obtain the power per unit solid angle (watts per square radian or steradian) or radiation intensity $U$. It should be noted that the radiation intensity is also independent on the radius (Rhodes, 1999). The radiation intensity can be expressed as:

$U(\theta, \phi)=U_{m}|F(\theta, \phi)|^{2}$

Where

$\mathrm{U}_{\mathrm{m}}$ denotes the maximum radiation intensity

$\mathrm{U}_{\mathrm{m}}=\mathrm{U}\left(\theta_{\max }, \phi\right.$ max $)$

$|F(\theta, \phi)|^{2}$ denotes the power pattern normalized to a maximum value of unity in direction $\left(\theta_{\max }, \phi\right.$ max $)$. The total power radiation is obtained by the radiation intensity over all angles around the antenna:

$P=\iint U(\theta, \phi) d \Omega=U_{m} \iint|F(\theta, \phi)|^{2} d \Omega \quad 3.0$

Where

$\mathrm{d} \Omega$ denotes $\operatorname{Sin} \theta d \theta d \phi$

Antenna Gain: It is a measure of how efficient the antenna transforms available power at its input terminals to radiated power, together with its directivity properties (Joe and John, 2005). The gain can be expressed as

$G(\theta, \phi)=\frac{4 \pi U(\theta, \phi)}{P_{i n}}$

Where $\mathrm{G}(\theta, \phi)$ denotes the gain, $\mathrm{U}(\theta, \phi)$ denotes radiation intensity of antenna in direction of $(\theta, \phi)$ including the effects of any losses on the antenna and $\mathrm{P}_{\text {in }}$ denotes the input power absorbed by the antenna.

However in practice, input power experience losses, which may be due to absorption of nearby structures or loss of power with the obstruction of line of sight (LOS). And radiation efficiency $\left(\mathrm{e}_{\mathrm{r}}\right)$ of an antenna is given as:
$\mathrm{e}_{\mathrm{r}}=\frac{P}{P_{\text {in }}} \quad 0 \leq \mathrm{e}_{\mathrm{r}} \geq 1$

\section{0}

Therefore, the maximum gain without the effects of mismatches of impedance or polarization is computed by obtaining the product of the directional characteristic of maximum directivity gain $D(\theta, \phi)$ multiplied by radiation efficiency, via:

$G(\theta, \phi)=e r \frac{4 \pi U(\theta, \phi)}{P}=\operatorname{erD}(\theta, \phi)$

Antenna Polarization: Polarization of an antenna is the polarization of the wave radiated in a given direction by the antenna when transmitting or receiving. Thomas (2005) classified the types of polarization as: vertical linear, horizontal linear, lefthand circular, right-hand circular, left-hand circular elliptical and right-hand elliptical. Polarization of an uniform plane wave describes the shape and locus of the tip of the Electric field $E(z, t)$ (in the plane orthogonal to the direction of propagation) as a function of time. The electric field phasor consists of $x$ and y component.

$\tilde{E}(z)=\hat{x} \tilde{E}_{x}(z)+\hat{y} \tilde{E}_{y}(z)$

Where

$\tilde{E}_{x}(z)=\tilde{E}_{x o} e^{-j k z}$ and $\tilde{E}_{\mathrm{xo}}$ is complex amplitudes

$\tilde{E}_{y}(z)=\tilde{E}_{y o} e^{-j k z}$ and $\tilde{E}_{\mathrm{yo}}$ is complex amplitudes

$z$ denotes the propagation direction

Besides that, wave polarization depends on the phase of $E_{y o}$ relative to that of $E_{x o}$, but not on the absolutes phases of $E_{x o}$ and $E_{y 0}$. A wave is linearly polarized if $\operatorname{Ex}(z, t)$ and $\operatorname{Ey}(z, t)$ are in phase $(\delta=0)$ or out of phase $(\delta=\pi)$ (Thomas $A, 2005)$. If $\delta$ is nonzero, then axial ratio is finite. When $\delta>0, E_{y}$ leads $E_{x}$ in phase, and the sense of rotation is left and conversely if $\delta<0$, the sense is right-hand.

Phasor electric field expression can be expressed as:

$E(z)=\left(E_{x} \hat{x}+E_{y} e^{j \bar{a}} \hat{y}\right) e^{-j k z}$ 
Equations 9.0 and 10.0 are the equations for instantaneous electric field and the modulus of the intensity and the direction of the electric field propagated respectively.

$$
E(x, t)=E_{x}(\cos \omega t-k z) \hat{x}+E_{y} \cos (\omega t-k z+\delta) \hat{y}
$$

And

$$
\begin{gathered}
|E(z, t)|=\sqrt{E_{x}^{2} \operatorname{Cos}^{2}(\omega t-k z)+E_{y}{ }^{2} \operatorname{Cos}^{2}(\omega t-k z+\delta)^{2}} \\
10.0
\end{gathered}
$$

The electric field $E(z, t)$ can be defined in the $x-y$ plane at a specific $z$ with respect to the zero-phase reference component of $E(z, t)$ as the inclination angle.

$$
\psi=\tan ^{-1}\left(\frac{E_{y}(z, t)}{E_{x}(z, t)}\right)
$$

Voltage Standing Wave Ratio (VSWR): VSWR is a measurement of the mismatch between two transmission lines. It provides a measurement of the amount of signal being reflected back from the mismatch, which is directly related to the amount of energy that is transmitted (Gary M. 1996). VSWR computed as the ratio of the net power to the forward power and can be expressed as the ratio of maximum to minimum voltage on the transmission line. The VSWR can be expressed as:

$V S W R=\frac{V_{\max }}{V_{\min }}=\frac{V_{i n c}+V_{r e f l}}{V_{i n c}-V_{\text {refl }}}$

Where $V_{\max }$ is the maximum voltage on the transmission line (feed cable), $V_{\min }$ is the minimum voltage on the transmission line, $V_{\text {inc }}$ is the magnitude of the incident wave, and $V_{\text {refl }}$ is the magnitude of the reflected wave. The reflection coefficient $\rho$ is the ratio of reflected to incident waves and is given by

$$
\rho=\frac{V_{-}}{V_{+}}
$$

or, in terms of impedance,
$\rho=\frac{Z_{L}-Z_{o}}{Z_{L}+Z_{o}}$

Where $V_{+}$is the incident wave (magnitude and phase), $V_{-}$is the reflected wave (magnitude and phase), $Z_{o}$ is the characteristic impedance of the transmission line (magnitude and phase), and $Z_{L}$ is the impedance of the load line (magnitude and phase).

If the load impedance is equal to the characteristic impedance of the transmission line, the reflection coefficient would be zero because there is no mismatch in this case. In addition, unlike VSWR, the reflection coefficient has both magnitude and phase. The magnitude of the reflection coefficient is then

$$
|\rho|=\frac{V_{\text {refl }}}{V_{\text {inc }}}=\frac{V S W R-1}{V S W R+1}
$$

The transmission coefficient $\mathrm{T}$ is defined as the ratio of transmitted to incident waves and is given by

$\tau=\frac{V_{L}}{V_{+}}$

or, in terms of impedance,

$$
\tau=\frac{2 Z_{o}}{Z_{L}+Z_{o}}
$$

Where $V_{L}$ is the wave transmitted through the mismatch to the load side (magnitude and phase). The ratio of the reflected power to the incident power is given as

$$
\frac{P_{r e f l}}{P_{i n c}}=\frac{V_{r e f l}^{2}}{V_{i n c}^{2}}=|\rho|^{2}
$$

So that $P_{\text {net }}=P_{\text {inc }}-P_{\text {refl }}$

This can be expressed in a VSWR correction factor given in $\mathrm{dB}$ as 
$C_{V S W R}=10 \log _{10}\left(\frac{4 V S W R}{(V S W R+1)^{2}}\right)$

The VSWR component covered here is not the only antenna VSWR term related to antenna measurements. If an antenna is not in a free-space environment, energy reflected back from other objects will affect the VSWR measurement. However, this term is a measure of the antenna's interaction with its environment rather than a measurement of an inherent property of the antenna.

Yagi Uda Antenna Design Parameter: According to Labade and Deosarkar (2010), one cannot always optimize the performance to get the best Directivity, Front-to-back ratio and input impedance at a particular frequency, as these parameters are complicated functions of element radii, dimensions and relative spacing because of the mutual coupling among the dipoles. Therefore designs are based on the trade-off so that a weighted combination of
Directivity, Front-to back ratio and input impedance needs to be optimized

A Yagi antenna develops an end fire radiation pattern and for optimum gains the reflector and driven elements are spaced 0.25 to 0.354 wavelengths, director-to-director spacing is 0.15 to 0.25 wavelengths apart. Reflector length is typically 0.5 wavelengths to 1.05 that of the driven element. The driven element is calculated at resonance without the presence of parasitic elements. The directors are 10 to $20 \%$ shorter than driven elements at resonance (Adedokun et.al, 2010).

For this work, boom length is fixed at $1.11 \lambda$ and the directors varied in length in a random fashion from $0.25 \lambda$ to $0.4 \lambda$ with an average spacing of less than $0.1 \lambda$. In practice, the Yagi-Uda antenna often consists of one reflector and two or more directors to provide better gain characteristics. To carry out the design of the antenna, the following parameters were needed. The typical design parameters of VHF are given below.

Table 1.0: Design Parameters of a Yagi Uda Antenna

\begin{tabular}{|l|l|l|l|l|l|}
\hline $\begin{array}{l}\text { Number of } \\
\text { Elements }\end{array}$ & $\begin{array}{l}\text { Length of } \\
\text { Reflector }\left(L_{R}\right) \mathbf{m}\end{array}$ & $\begin{array}{l}\text { Length of Driven } \\
\text { Element }(\mathbf{L}) \mathbf{m}\end{array}$ & $\begin{array}{l}\text { Length of } \\
\text { Director }\left(L_{D}\right) \mathbf{m}\end{array}$ & $\begin{array}{l}\text { Spacing of } \\
\text { Reflector }\left(\mathbf{S}_{R}\right) \mathbf{m}\end{array}$ & $\begin{array}{l}\text { Spacing } \\
\text { Director }\left(\mathbf{S}_{\mathrm{D}}\right) \mathbf{m}\end{array}$ \\
\hline 12 & $0.51 \lambda$ & $0.5 \lambda$ & $0.456 \lambda$ & $0.25 \lambda$ & $0.15 \lambda$ \\
\hline
\end{tabular}

In addition, the antenna design targets the field strength in the main beam to be larger than a critical minimum value, the directivity be large enough over the whole frequency band, the antenna's input impedance match to the generator reasonably well over the entire band and that the VSWR be small. In

Table 2.0: Calculated Values of the Antenna Dimensions

(cm)

\begin{tabular}{|c|c|c|c|c|c|c|}
\hline $\mathrm{L}_{\mathrm{R}}(\mathrm{cm})$ & L (cm) & $L_{D}(\mathrm{~cm})$ & $\mathrm{S}_{\mathrm{R}}(\mathrm{cm})$ & $\mathrm{S}_{\mathrm{D}}(\mathrm{cm})$ & Tube diameter $(\mathbf{c m})$ & Boom Length (m) \\
\hline 70.70 & 69.30 & 63.20 & 34.70 & 20.80 & 0.80 & 2.843 \\
\hline
\end{tabular}

Selecting Optimal Values for Antenna Dimension: The gain of Yagi-Uda antenna depends heavily on the number and length of directors. The gain can be increased by increasing the number of director elements while the operating frequency can be increased by reducing the lengths of all the elements. If increased bandwidth or specified input impedance is required, it is necessary to simulate this antenna repeatedly with variations in the element spacing and length, and to optimize for the specific requirement. In a work by Adedokun et.al, 2010, it noted that this paper, Nigerian Television Authority, Makurdi Network parameters are used to simulate optimal dimensions of the antenna. It transmits at VHF, operating on Channel $13(215$ - 218) MHz. Using the aforementioned conditions and Table 1.0, the calculated values for the design dimensions for the antenna is tabulated on Table 2.0.

forward gain can be increased by adding the directors of a Yagi Uda.

To optimize the 12 elements Yagi Uda antenna, the spacing between directors was varied while holding the reflector exciter spacing and lengths of all elements constant. The best results gave directivity of $13.2 \mathrm{dBi}$. For an experiment that maintained the length parameters and varies the spacing gives directivity of $13.9 \mathrm{dBi}$. Best results were achieved when there is both spacing and length perturbation of the antenna array. The initial gain is $13.9 \mathrm{dBi}$ but the computation returned directivity value of $14.9 \mathrm{dBi}$ after varying both the spacing and length of the array. 
Table 3.0 shows the final results with the highest gain achieved.

Table 6.0: Directivity optimization for 12 Eelement Yagi-Uda array for VHF band atr $=0.15 \mathrm{~cm}$

\begin{tabular}{|l|l|l|l|}
\hline \multicolumn{1}{|c|}{$\begin{array}{l}\text { Dipole } \\
\text { Length }\end{array}$} & $\begin{array}{c}\text { Initial } \\
\text { Array }\end{array}$ & $\begin{array}{c}\text { Array after } \\
\text { Spacing }\end{array}$ & $\begin{array}{c}\text { Optimum Array } \\
\text { after } \\
\text { Spacing and } \\
\text { Length } \\
\text { Perturbation }\end{array}$ \\
\hline $\mathrm{L}_{\mathrm{D}}(\mathrm{cm})$ & 73.50 & 73.50 & 79.50 \\
\hline $\mathrm{L}_{(\mathrm{cm})}$ & 69.30 & 69.30 & 76.78 \\
\hline $\mathrm{L}_{\mathrm{D} 1}(\mathrm{~cm})$ & 61.10 & 61.10 & 74.19 \\
\hline $\mathrm{L}_{\mathrm{D} 2}(\mathrm{~cm})$ & 61.10 & 60.70 & 64.00 \\
\hline $\mathrm{L}_{\mathrm{D} 3}(\mathrm{~cm})$ & 60.50 & 60.50 & 64.30 \\
\hline $\mathrm{L}_{\mathrm{D} 4}(\mathrm{~cm})$ & 60.50 & 60.40 & 63.30 \\
\hline $\mathrm{L}_{\mathrm{D} 5}(\mathrm{~cm})$ & 60.40 & 60.50 & 62.50 \\
\hline $\mathrm{L}_{\mathrm{D} 6}(\mathrm{~cm})$ & 56.60 & 60.40 & 62.30 \\
\hline $\mathrm{L}_{\mathrm{D} 7}(\mathrm{~cm})$ & 56.60 & 60.40 & 59.40 \\
\hline $\mathrm{L}_{\mathrm{D} 8}(\mathrm{~cm})$ & 55.20 & 56.60 & 58.80 \\
\hline $\mathrm{L}_{\mathrm{D} 9}(\mathrm{~cm})$ & 55.20 & 55.20 & 58.00 \\
\hline $\mathrm{L}_{\mathrm{D} 10}(\mathrm{~cm})$ & 54.10 & 54.10 & 56.66 \\
\hline $\mathrm{Directivity}_{1}$ & 13.9 & $13.9 \mathrm{dBi}$ & $14.9 \mathrm{dBi}$ \\
\hline & & & \\
\hline & &
\end{tabular}

\section{RESULTS AND DISCUSSIONS}

Antenna Magus 2.2 software which is a Windowscompatible antenna analysis, modeling and design software package was used to analyze the best dimensions selected. This allows simulation of the impedance and patterns of the antenna dimension designed.

Antenna Simulation Results: The best performance of the simulated results was obtained when both the director spacing and all the elements length were varied. The result of the simulation of the gain against frequency after the perturbation is as shown on Figure 1.0.

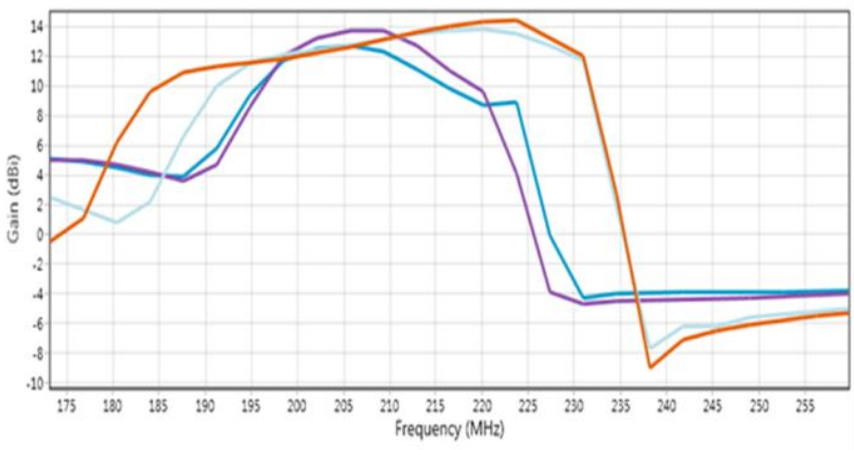

Fig 1.0 Graph of Gain plotted against Frequency after Perturbation of director spacing and all element lengths

From the graph in Figure 1.0, it is observed that there was a significant increment in the total gain of the antenna (from $13.75 \mathrm{dBi}$ at $220.1 \mathrm{MHz}$ to $14.37 \mathrm{dBi}$ at 223.7 MHz which is indicated by the red line on the graph) with a corresponding $18.04 \%$ increase in the bandwidth of the antenna. Increase in both gain and bandwidth is an advantage for the antenna.

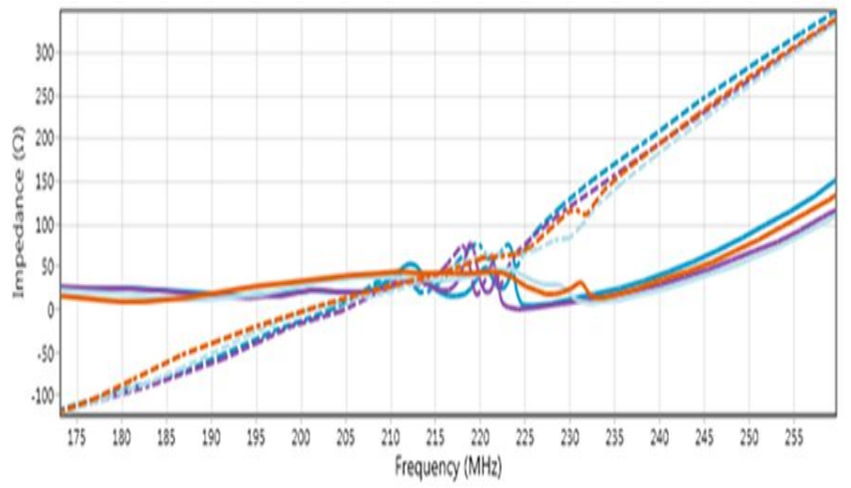

Fig 2.0: Graph of Impedance plotted against Frequency after Perturbation of director spacing and all element lengths

Figure 2.0 shows that the peak real impedance of the antenna is at $134.61 \Omega$ which corresponds to frequency of $259.7 \mathrm{MHz}$ (the thick red line on the graph) and the Real impedance at zero-crossing frequency increased to $334.0 \Omega$ at Frequency 200.9 $\mathrm{MHz}$ compared to simulation of other dimensions. 


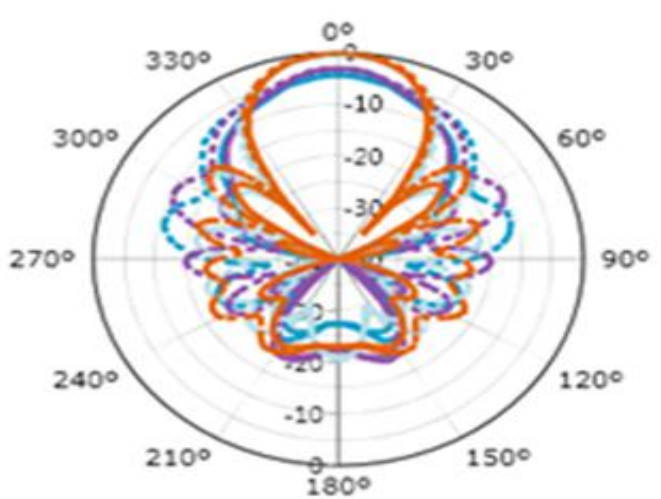

Fig 3.0: Radiation pattern at the Centre frequency after Perturbation of director spacing and all element lengths

From the radiation Pattern in Figure 3.0, it can be seen that the peak gain at angle (freq) $\varphi=0^{0}$ is 14.03 $\mathrm{dBi}$ and the peak gain at angle (freq) $\varphi=90^{\circ}$ is 10.96 $\mathrm{dBi}$ where $\theta=0^{0}(216.5 \mathrm{MHz})$. This is a significant improvement compared to other design dimensions for the antenna.

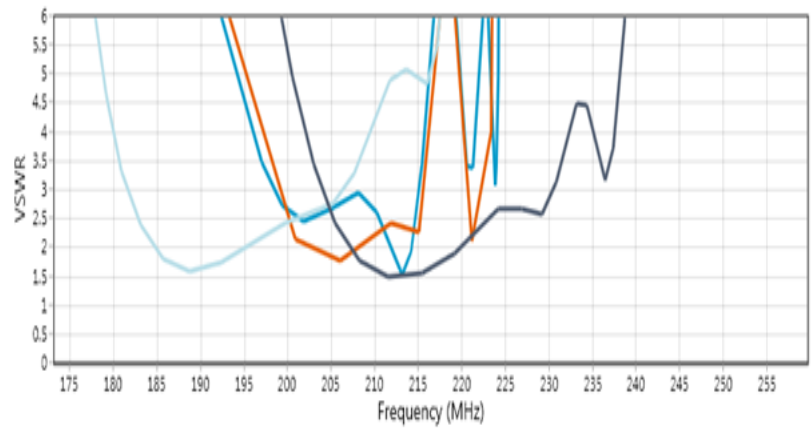

Fig 4.0: Graph of VSWR verses Frequency after Perturbation of director spacing and all element lengths

Figure 4.0 is the graph of the best VSWR against Frequency from the various simulations. It shows that the frequency at which VSWR is lowest (VSWR = 1.463) is now at frequency of 212.8 as against the next best at VSWR $=1.572$ at $189.0 \mathrm{MHz}$. These results show a significant reduction in the value of VSWR which is a good for the antenna.

Conclusion : The design of the Yagi Uda antenna for the VHF band has been presented. The design and simulation is done using simulation software know as Antenna Magus 2.2. The correlation of the simulated results with the objectives shows that the Bandwidth coverage from $185 \mathrm{MHz}$ to $237 \mathrm{MHz}$ had been covered for the Yagi Uda antenna. This bandwidth is quite satisfactory compared to the terrestrial TV frequency band. The simulation gives a unique set of Yagi antenna dimensions which best satisfy optimization goals, but it does not optimize antenna performances over the wider statistical distribution of antenna dimensions. This will make the antenna design very narrow, sensitive and critical.

\section{REFERENCES}

1. Adedokun, A. and Adegoye, K. A. (2010): Development of $50 \mathrm{~dB}$ Yagi-Uda antenna for effective communication. Pacific Journal of Science and Technology. 11(1): 16-23.

2. Barclay, L.W. (1997): Radio System Parameters. Lancaster University: Lancaster, UK.

3. Bemani, M and Nikmehr, S. (2009): A Novel Wide-band Microstrip Yagi-Uda array antenna for WLAN Applications. Progress in Electromagnetics Research. 16: 389-406.

4. Cheng, D. K. (1991): Gain Optimization for YagiUda Arrays. IEEE Antennas and Propagation Magazine. 33(3): 42-45.

5. Gary M. M. (2004): Modern Electronic Communication, 6th Edition, New Jersey: Prentice Hall.

6. Goudos, S. K.; Siakavara, K.; Vafiadis, E. E. and Sahalos, J. N. (2010): Pareto optimal Yagi-Uda Antenna Design Using Multi-objective Differential Evolution. Progress In Electromagnetics Research. 105: 231-251.

7. Joe, C. and John, D. (2000): Newness Radio and RF Engineer's Pocket Book (Second Edition), Oxford Press.

8. Labade, R. P. and Deosarkar, S. B. (2010): Design of Yagi-Uda Antenna at $435 \mathrm{MHz}$ for Indian MST Radar International. Journal of Advanced Engineering and Application. 1: 239242.

9. Lohn, J. D.; Kraus, W. F.; Linden, D. S. and Colombano, S. P. (2001): Evolutionary Optimization of Yagi-UdaAntennas. Proceedings of Fourth Conference on Evolvable System, Tokyo. October 3-5, 2001: 236-243.

10. Perambur, S. and Rajeswari, C (2003): Antennas for Information Super Skyways: An Exposition on Outdoor and Indoor Wireless Antennas. Research Studies Press Ltd.

11. Rhodes, J. E. (1999): Antenna Handbook: US Marine Corps. PCN 144000006200 www.doctrine.quantrico.usme.mil.

12. Sun, B. H.; Zhou, S. G.; Wei, Y. F. and Liu, Q. Z. (2010): Modified Two-Element Yagi-Uda Antenna with Tunable Beams. Progress In Electromagnetics Research. 100: 175-187. 